\title{
Influence of gastrointestinal parasitism on biochemical variables in blood of laying hens
}

\section{Influencia del parasitismo gastrointestinal en las variables bioquímicas en la sangre de las gallinas ponedoras}

\author{
Josué P Topázio, ${ }^{1}$ M.Sc, Gabriela Campigotto, ${ }^{1}$ M.Sc, Marcel M Boiago, ${ }^{1}$ Ph.D, \\ Gustavo Machado, ${ }^{2}$ M.Sc, Diovani Paiano, ${ }^{1}$ Ph.D, Alexandre A Tonin, ${ }^{3}$ Ph.D, \\ Aleksandro S da Silva, ${ }^{1 *}$ Ph.D.
}

\begin{abstract}
${ }^{1}$ Universidade do Estado de Santa Catarina (UDESC), Departamento de Zootecnia, Rua Beloni Trombita Zanini, 680E - Santo Antônio, Chapecó - SC, 89815-630, Brasil. ${ }^{2}$ Universidade Federal do Rio Grande do Sul (UFRGS), Laboratório de Epidemiologia Veterinária, Porto Alegre - RS, 91540-000, Brasil. ${ }^{3}$ Universidade Federal de Santa Maria (UFSM), Departamento de Microbiologia e Parasitologia, Avenida Roraima, 1000 - Camobi, Santa Maria - RS, 97105-900, Brasil. *Correspondence: aleksandro_ss@yahoo.com.br
\end{abstract}

Received: October 2014; Acepted: February 2015.

\begin{abstract}
Objective. This study describes the influence of endoparasites and diet on biochemical variables in sera of brown laying hens. Materials and methods. Biochemical parameters (seric levels of cholesterol, triglycerides, total protein, uric acid, albumin, and alanine aminotransferase activity) were assessed in blood samples of poultry. Three populations of laying hens of different properties $(n=20$ each) represented our experimental design, from which blood (serum) and feces were collected for biochemical and parasitological analyzes, respectively. Results. From 60 feces samples evaluated 49 were positive for the presence of parasites and 11 were negative. Among the positive samples, 23 had infection with Eimeria spp and the other 26 samples had mixed parasitism with Eimeria spp and Heterakis gallinarum, which influenced (increasing) the levels of total protein and globulin. However, when parasites were analyzed separately, it was observed that the infection by Eimeria spp affected parameters such as cholesterol (which increased, $p=0.001$ ) and triglycerides, showing lower results than when the hen was parasitized by Eimeria spp. $(p=0.01)$. Significant $(p<0.05)$ difference was observed in all biochemical variables when the three diets were compared. Conclusions. Our results allowed concluding that the parasitic infection and diet in laying hens influenced the results of serum biochemical parameters evaluated. The main conclusion we found that parasitism caused an increase in total protein and globulin (ie immune response), and there was a negative correlation between parasite load and albumin, uric acid and ALT.
\end{abstract}

Key words: Biochemical variables, Eimeria, Heterakis gallinarum, poultry (Source: CAB, MeSH).

\section{RESUMEN}

Objetivo. Este estudio describe la influencia de la dieta sobre los endoparásitos y variables bioquímicas en el suero de las gallinas ponedoras marrones. Materiales y métodos. Los parámetros bioquímicos (niveles séricos de colesterol, triglicéridos, proteínas totales, ácido úrico, albúmina, y la actividad de la alanina aminotransferasa) se evaluaron en muestras de sangre de las aves de corral. Tres 
poblaciones de gallinas ponedoras de distintas propiedades ( $n=20$ cada uno) representan nuestro diseño experimental, de la que se recogieron la sangre (suero) y las heces de bioquímica y análisis parasitológico, respectivamente. Resultados. De 60 muestras de heces evaluadas, 49 fueron positivos para la presencia de parásitos y 11 fueron negativos. Entre las muestras positivas, 23 tenían infección con Eimeria spp y las otras 26 muestras tenían parasitismo mezclado con Eimeria spp y Heterakis gallinarum, que influyó (aumentando) los niveles de proteína total y globulina. Sin embargo, cuando se analizaron por separado los parásitos, se observó que la infección por Eimeria spp afectó parámetros tales como el colesterol (el cual se incrementó $p=0.001$ ) y los triglicéridos, que mostraron resultados más bajos que cuando las gallinas fueron parasitadas por Eimeria spp. $(p=0.01)$. Se observó diferencia $(p<0.05)$ en todas las variables bioquímicas, cuando se compararon las tres dietas. Conclusiones. Nuestros resultados permitieron concluir que la infección parasitaria y la dieta de las gallinas ponedoras influyeron en los resultados de los parámetros bioquímicos séricos evaluados. La principal conclusión que se encontró es que el parasitismo causó un aumento de la proteína total y la globulina (es decir, la respuesta inmune), y hubo una correlación negativa entre la carga de parásitos y albúmina, ácido úrico y ALT.

Palabras clave: Aves de corral, variables bioquímicas, Eimeria, Heterakis gallinarum. (Fuente: CAB, $\mathrm{MeSH}$ ).

\section{INTRODUCTION}

Sanity and nutrition are essential factors for the proper animal development, especially in production systems. Improper handling may be responsible for the occurrence of various diseases, including those caused by parasites, such as coccidiosis (1). Coccidiosis is caused by several species of the genus Eimeria, a protozoan. These intracellular parasites usually infect the cells of the digestive tract, causing cell disruption and tissue injury due to the parasite multiplication, causing, consequently occurs bleeding, leading to malabsorption of nutrients and being a gateway to other pathogens $(1,2)$. Broilers, layers and breeders in general are subjected to contamination with Eimeria spp., mainly because in different breeding systems the animals are infected by ingesting sporulated oocysts in water and laying bed. Several species of Eimeria can infect chickens, but the most commonly found are $E$. acervulina, E. brunetti, $E$. maxima, E. mitis, E. necatrix, E. praecox and $E$. tenella (3). Ascaris galli and Heterakis gallinarum are also endoparasites commonly observed infecting chickens. These nematodes normally infect the large intestine of these animals, causing weight loss, diarrhea and intestinal mucosal lesion $(2,4)$. This infection usually leads to irreparable intestine damage, affecting the absorption of nutrients (2).

The analysis of biochemical variables can assist in a rapid diagnosis. Among these variables, the most relevant to chickens is alanine aminotransferase (ALT), a catalytic enzyme that has the function of transaminase and acts in the catabolism of amino acids and transport of nitrogen among organs (5). In this sense, ALT activity is an indicator of liver and muscle injuries,

\section{INTRODUCCIÓN}

La higiene y la nutrición son factores esenciales para el desarrollo adecuado del animal, especialmente en los sistemas de producción. El manejo inadecuado puede ser el responsable de la aparición de algunas enfermedades, incluyendo aquellas causadas por parásitos, como es el caso de la coccidiosis (1). La coccidiosis es causada por varias especies del género Eimeria, un protozoario. Estos parásitos intracelulares generalmente infectan las células del tracto digestivo, causando trastornos celulares y lesiones en los tejidos debido a la multiplicación de los parásitos, presentándose como consecuencia hemorragia, lo que conduce a la mala absorción de nutrientes y se convierte en una puerta de entrada para otros patógenos $(1,2)$. Los pollos de engorde, las ponedoras y las reproductoras, están sometidos en general a la contaminación con Eimeria spp., principalmente porque en los diferentes sistemas de cría los animales se infectan por la ingestión de ooquistes esporulados presentes en el agua y en los nidos. Algunas especies de Eimeria pueden infectar a las gallinas, pero las que se encontraron más comúnmente fueron $E$. acervulina, $E$. brunetti, $E$. maxima, E. mitis, E. necatrix, E. praecox y E. tenella (3). El Ascaris galli y el Heterakis gallinarum son también endoparásitos que comúnmente infectan a las gallinas. Estos nematodos normalmente infectan el intestino grueso de estos animales, causando pérdida de peso, diarrea y lesión de la mucosa intestinal $(2,4)$. Esta infección por lo general conduce a un daño irreparable del intestino, lo que afecta la absorción de nutrientes (2).

El análisis de las variables bioquímicas puede ayudar a un diagnóstico rápido. Entre estas variables, la más relevante para las gallinas es la alanina aminotransferasa (ALT), una enzima catalítica que tiene la función de la transaminasa y actúa en el 
especially when it is significantly increased (6). Other relevant metabolites that can be assessed are the levels of total protein, albumin and globulin, proteins that can be influenced by the type of diet and creation system, as well as by the different infectious diseases due to the involvement of globulins in the inflammatory response $(2,7)$. Yet, the levels of cholesterol and triglycerides may reflect eating disorders, often derived from the diet (8).

Considering that the levels of some blood biochemical variables are linked to the physiological condition and nutritional profile of the animal, as well as it is known that eimeriosis may influence directly the functional animal homeostasis $(4,9)$, this study aimed to evaluate the effects of parasitic diseases and diet on blood biochemical parameters of laying hens.

\section{MATERIAIL AND METHODS}

Ethical aspects. The project was approved by the ethics committee on animal research at the Universidade do Estado de Santa Catarina, protocol number, 01.24.14.

Study sites. Samples were collected from three commercial poultry farms located in different towns of western Santa Catarina State, southern Brazil. The hens were housed collectively in a shed, and the animals were laying eggs in nests.

Animals and collection of samples. Our experimental design used 60 laying hens of Hy Line lineage, aging between 17 and 23 weeks. From each property it was collected a total of 20 blood and faeces samples.

For collect the samples the laying hens were manually contained. By brachial vein route, using a $5.0 \mathrm{~mL}$ syringe and needle $25 \times 7$, it was collected an average of $2.5 \mathrm{~mL}$ of blood, from which sera samples were obtained through blood centrifugation at $3500 \mathrm{rpm}$ for 10 minutes. Serum samples were identified and stored at $-20^{\circ} \mathrm{C}$ until the biochemical analyzes.

Samples of feces were collected immediately after collection of blood through the direct collect of cloacal feces ( 2 to $4 \mathrm{~g}$ ). They were stored in plastic containers and kept under refrigeration $\left(10^{\circ} \mathrm{C}\right)$ until laboratorial processing.

Biochemical variables. Seric levels of cholesterol, triglycerides, total protein, uric acid, albumin and alanine aminotransferase (ALT) activity were assessed using a semi-automated analyzer (Bioplus $2000^{\circledR}$ ), and used specific kits catabolismo de los aminoácidos y en el transporte de nitrógeno entre los órganos (5). En este sentido, la actividad de la ALT es un indicador de lesiones hepáticas y musculares, en especial cuando aumenta significativamente (6). Otros metabolitos relevantes que se pueden evaluar son los niveles de proteína total, albúmina y globulina, proteínas que pueden ser influenciadas por el tipo de dieta y el sistema de creación, así como por las diferentes enfermedades infecciosas debido a la participación de las globulinas en la respuesta inflamatoria $(2,7)$. Sin embargo los niveles de colesterol y triglicéridos pueden reflejar trastornos en la alimentación, a menudo derivados de la dieta (8).

Considerando que los niveles de algunas variables bioquímicas de la sangre están relacionados con las condiciones fisiológicas y el perfil nutricional del animal, se sabe que la eimeriosis puede influir directamente en la homeostasis funcional de los animales $(4,9)$; este estudio tuvo como objetivo evaluar los efectos de las enfermedades parasitarias y de la dieta sobre los parámetros bioquímicos de la sangre de las gallinas ponedoras

\section{MATERIALES Y MÉTODOS}

Aspectos éticos. El Proyecto fue aprobado por el comité de ética para la investigación con animales de la Universidade do Estado de Santa Catarina, protocolo número 01.24.14.

Sitios de estudio. Las muestras se tomaron en tres granjas comerciales de aves de corral localizadas en diferentes pueblos del oeste del estado Santa Catarina, en el sur de Brasil. Las gallinas se ubicaron colectivamente en un cobertizo y estas ponían sus huevos en nidos.

Animales y toma de muestras. En nuestro diseño experimental se utilizaron 60 gallinas del linaje Hy Line, con edades entre 17 y 23 semanas. En cada granja se recogieron un total de 20 muestras de sangre y de heces.

Para la recolección de las muestras las gallinas ponedoras fueron sostenidas manualmente. Por la vena braquial, mediante una jeringa de $5.0 \mathrm{ml}$ y una aguja $25 \times 7$, se recogió una media de $2.5 \mathrm{ml}$ de sangre y se obtuvieron muestras de suero mediante centrifugación de la sangre a 3500 rpm durante 10 minutos. Las muestras de suero se identificaron y se almacenaron a $-20^{\circ} \mathrm{C}$ hasta la realización del análisis bioquímico.

Las muestras de la materia fecal se tomaron inmediatamente después de la recolección de la sangre mediante toma directa de las heces cloacales (de 2 a $4 \mathrm{~g}$ ). Estas se almacenaron en recipientes 
for each test (Gold Analisa Diagnóstica ${ }^{\circledR}$ Ltda). Globulin levels were calculated as the difference between total protein and albumin (10).

Parasitological of faeces. Fecal samples $(1 \mathrm{~g})$ were processed by the flotation method (centrifugal flotation technique), using sucrose supersaturated solution $(15 \mathrm{~mL})$. The samples were centrifuged at $2500 \mathrm{rpm}$ for 10 minutes. The microscopic evaluation aimed to visualize eggs, cysts and oocysts of parasites in 100 and 400x, as well as quantified the number of oocysts per gram of feces (OOPG) of coccidian in glass slide each. The helminth eggs were only identified, and not quantified.

Diet. In each farm, two samples $(500 \mathrm{~g})$ of ration were collected and identified. These samples were kept under refrigeration condition throughout the transport from the site of analysis, where they were stored frozen until analysis. The samples were ground in a hammer mill type using sieves of $1 \mathrm{~mm}$, and then conducted analysis of dry matter, crude protein, gross energy, ether extract and mineral matter (11), of the agreement with the methodology (12).

Statistical analysis. The data from biochemical analysis: albumin, uric acid, ALT, cholesterol, triglycerides, total protein and globulin, as well as the OOPG count were first analyzed by descriptive statistics for contingency of the information and for further assumptions and what is presented as descriptive is the mean and standard deviation. All above parameters were tested for normality of variance by ShapiroWilk test, skewness, kurtosis and homogeneity by Levene's test previously to ANOVA analysis. The OOPG, alt, cholesterol, triglycerides, total protein and globulin were transformed by $\left\{\log _{10}(x+1)\right\}$ before linear and variance analysis. All biochemical parameters were also analyses for correlation with OOPG count by Kendall rank correlation. For parametric analysis first type of diet was analyzed by compering its influence on biochemical parameter using one-way ANOVA as well as linear regression model for correlation, where necessary (statistic difference were found), Bonferroni's test (13). Finally, it was also analyzed the influence of parasite infection type on the biochemical parameters by compering its influence on biochemical parameter using one-way ANOVA as well as linear regression model for correlation, where necessary (statistic difference were found), Bonferroni's test (13). It was considered significantly different when $p<0.05$. The whole statistical process was carried out with R-language, v.2.15.2 (R Development Core Team, 2012). plásticos y se mantuvieron refrigeradas $\left(10^{\circ} \mathrm{C}\right)$ hasta cuando se procesaron en el laboratorio.

Variables bioquímicas. Los niveles séricos de colesterol, triglicéridos, proteína total, ácido úrico, albúmina y de actividad de la alanina aminotransferasa (ALT) se evaluaron utilizando un analizador semiautomático (Bioplus 2000 ${ }^{\circledR}$ ); se utilizaron kits específicos para cada prueba (Gold Analisa Diagnóstica ${ }^{\circledR}$ Ltda.). Los niveles de globulina se calcularon como la diferencia entre proteína total y albúmina (10).

Parasitológico de las heces. Las muestras fecales $(1 \mathrm{~g})$ se procesaron por el método de flotación (técnica de flotación centrífuga) utilizando una solución supersaturada de sacarosa (15 mL). Las muestras se centrifugaron a $2500 \mathrm{rpm}$ durante 10 minutos. La evaluación microscópica permitió visualizar huevos, quistes y ooquistes de parásitos a 100x y 400x, e igualmente se cuantificó el número de ooquistes de coccidios por gramo de heces (OOPG) en cada portaobjetos de vidrio. Los huevos de los helmintos solamente se identificaron mas no se cuantificaron.

Dieta. En cada granja se recogieron y se identificaron dos muestras $(500 \mathrm{~g}$ ) de la ración. Estas muestras se guardaron bajo condiciones de refrigeración durante todo su transporte desde el lugar del análisis, donde se almacenaron congeladas hasta el momento del análisis. Las muestras se molieron en un molino de martillos, se utilizaron tamices de 1 $\mathrm{mm}$ y luego se realizaron análisis de materia seca, proteína cruda, energía bruta, extracto de éter y contenido de minerales (11), del acuerdo con la metodología (12).

Análisis estadístico. Los siguientes son los datos del análisis bioquímico: albúmina, ácido úrico, ALT, colesterol, triglicéridos, proteína total y globulina, así como el recuento OOPG, se analizaron primero por estadística descriptiva para la contingencia de la información y para las nuevas hipótesis; lo que se presenta como descriptiva corresponde a las desviaciones media y estándar. A todos los parámetros anteriores se les realizaron pruebas de normalidad de varianza mediante el test de ShapiroWilk, asimetría, curtosis y homogeneidad mediante la prueba de Levene previo al análisis ANOVA. EI OOPG, ALT, colesterol, triglicéridos, proteína total y globulina se transformaron por $\left\{\log _{10}(x+1)\right\}$ antes del análisis lineal y de la varianza. A todos los parámetros bioquímicos también se les hizo el análisis de correlación con el recuento OOPG por rango de correlación según Kendall. Para el análisis paramétrico, se analizó el primer tipo de dieta comparando su influencia sobre los parámetros bioquímicos, utilizando ANOVA de una vía, (Análisis de Varianza, por sus siglas en inglés), así como 


\section{RESULTS}

Parasites and infection type. Among the samples tested, 11 were negative and 49 were positives for Eimeria spp. (oocysts) and/or Heterakis gallinarum (eggs). The OOPG showed a mean value of 596.46 (ranging between 0-9696) in the three properties under evaluation.

Diet. The data of diet analyzes are presented in table 1 . Numerically, the diet 1 had lower levels of crude protein, gross energy, ether extract, and mineral matter when compared with diets 2 and 3 .

Table 1. Composition of diets fed to laying hens on the three properties studied.

\begin{tabular}{lccc}
\hline Composition & Diet $^{\mathbf{1}}$ & Diet $^{\mathbf{2}}$ & Diet $^{\mathbf{3}}$ \\
\hline Dry matter (\%) & 88.26 & 88.48 & 88.87 \\
Crude protein (\%) & 16.62 & 17.05 & 18.49 \\
Gross energy (Mcal/Kg) & 2.60 & 2.80 & 2.85 \\
Ash (\%) & 12.45 & 13.2 & 13.04 \\
Crude fat (\%) & 4.3 & 4.7 & 4.8 \\
\hline
\end{tabular}

Relationship between biochemical variables and OOPG. Negative correlation between OOPG (Eimeria spp) and albumin $(r=-0.28)$, uric acid $(r=-0.28)$, and ALT $(r=-0.19)$ in the serum of the laying hens was observed (Table 2). It was not observed correlation between OOPG and cholesterol, triglycerides, total protein and globulin (Table 2).

Relationship between biochemical variables and diet. The results of biochemical variables in layers serum at three farms with different diets were shown in table 3 . There was statistical significance between diets and albumin levels $(F=7.62, d f=2, p<0.001)$ being higher on diets 2 and 3 , respectively $(B=0.50 \pm 0.13, t=3.74$,

Table 2. Correlation between the number of oocysts per gram of faeces (OOPG) of Eimeria spp. and serum biochemical variables (albumin, uric acid, alanine aminotransferase (ALT), cholesterol, triglycerides, total protein and globulin) in laying hens.

\begin{tabular}{lcc}
\hline Correlation of Kendall & Rho & P-value \\
\hline $\begin{array}{l}\text { OOPG } \\
\text { Albumin }(\mathrm{g} / \mathrm{dL})\end{array}$ & -0.28 & 0.002 \\
$\begin{array}{l}\text { OOPG } \\
\text { Uric acid }(\mathrm{mg} / \mathrm{dL})\end{array}$ & -0.28 & 0.001 \\
$\begin{array}{l}\text { OOPG } \\
\text { ALT }(\mathrm{U} / \mathrm{L})\end{array}$ & -0.19 & 0.03 \\
$\begin{array}{l}\text { OOPG } \\
\text { Cholesterol }(\mathrm{mg} / \mathrm{dL})\end{array}$ & 0.13 & 0.14 \\
$\begin{array}{l}\text { OOPG } \\
\text { Triglycerides }(\mathrm{mg} / \mathrm{dL})\end{array}$ & 0.07 & 0.39 \\
$\begin{array}{l}\text { OOPG } \\
\text { Total protein }(\mathrm{g} / \mathrm{dL})\end{array}$ & -0.03 & 0.37 \\
OOPG & & \\
Globulin $(\mathrm{g} / \mathrm{dL})$ & 0.18 & 0.08 \\
\hline
\end{tabular}

el modelo de regresión lineal para la correlación; debido a que se encontró una diferencia estadística se hizo necesaria la prueba de Bonferroni (13). Por último, también se analizó la influencia del tipo de infección parasitaria sobre los parámetros bioquímicos comparando su influencia sobre estos utilizando el método ANOVA de una vía, al igual que el modelo de regresión lineal para correlación; debido a que se encontró una diferencia estadística se hizo necesaria la prueba de Bonferroni (13). Se consideró significativamente diferente cuando $p<0.05$. Todo el proceso estadístico general se llevó a cabo utilizando el Lenguaje-R, v.2.15.2 (R Development Core Team, 2012).

\section{RESULTADOS}

Parásitos y tipo de infección. De las muestras examinadas, 11 dieron resultados negativos y 49 positivos para Eimeria spp. (ooquistes) y/o Heterakis gallinarum (huevos). Los OOPG mostraron un valor medio de 596.46 (con rango entre 0-9696) en las tres granjas que se evaluaron.

Dieta. La información de los análisis de las dietas se presenta en la tabla 1 . Numéricamente la dieta 1 tiene los niveles más bajos de proteína cruda, energía bruta, extracto de éter y contenido de minerales en comparación con las dietas 2 y 3 .

Relación entre las variables bioquímicas y OOPG. Se observó una correlación negativa entre OOPG (Eimeria spp) y albúmina $(r=-0.28)$, ácido úrico $(r=-0.28)$, and ALT $(r=-0.19)$ en el suero de las gallinas ponedoras (Tabla 2). No se observó correlación entre OOPG y colesterol, triglicéridos, proteína total y globulina (Tabla 2).

Table 3. Influence of diet (three properties) on blood biochemical variables (albumin, uric acid, alanine aminotransferase (ALT), cholesterol, triglycerides, total protein and globulin) in laying hens.

\begin{tabular}{|c|c|c|c|}
\hline \multirow[t]{2}{*}{ Variables } & \multicolumn{3}{|c|}{ Mean and standard deviation* } \\
\hline & Diet $^{1}$ & Diet $^{2}$ & Diet $^{3}$ \\
\hline Albumin ( $\mathrm{g} / \mathrm{dL})$ & $1.69 \pm 0.40^{b}$ & $2.19 \pm 0.46^{a}$ & $2.07 \pm 0.41^{\mathrm{a}}$ \\
\hline Uric acid (mg/dL) & $3.57 \pm 1.49^{b}$ & $7.00 \pm 2.18^{\mathrm{a}}$ & $7.44 \pm 2.070^{\mathrm{a}}$ \\
\hline ALT $(U / L)$ & $15.00 \pm 5.01^{b}$ & $30.10 \pm 15.58^{a}$ & $30.28 \pm 18.00^{\mathrm{a}}$ \\
\hline Cholesterol (mg/dL) & $207.4 \pm 66.4^{a}$ & $149.6 \pm 51.2^{\mathrm{b}}$ & $103.25 \pm 47.2^{b}$ \\
\hline Triglycerides (mg/dL) & $486.4 \pm 180.5^{b}$ & $443.1 \pm 107.2^{b}$ & $714.9 \pm 169.5^{a}$ \\
\hline Total protein $(\mathrm{g} / \mathrm{dL})$ & $4.74 \pm 1.06^{\mathrm{b}}$ & $4.11 \pm 0.72^{\mathrm{b}}$ & $7.04 \pm 1.46^{a}$ \\
\hline Globulin (g/dL) & $3.05 \pm 0.90^{\mathrm{b}}$ & $1.92 \pm 0.70^{c}$ & $4.97 \pm 1.52^{\mathrm{a}}$ \\
\hline
\end{tabular}


$\mathrm{p}<0.01$ and $\mathrm{B}=0.38 \pm 0.13, \mathrm{t}=2.84, \mathrm{p}<0.001)$. Also was observed a statistical significance between diets and uric acid levels $(F=23.9$, $\mathrm{df}=2, \mathrm{p}<0.001$ ) being higher on diet 2 and 3 , respectively $(B=3.42 \pm 0.61, t=5.60, p<0.01$ and $B=3.87 \pm 0.61, t=6.32, p<0.001)$. Other analysis with statistical significance was between diets and ALT levels $(F=10, d f=2, p<0.001)$ being higher on diet 2 and 3 , respectively $(B=0.60 \pm 0.15$, $t=3.90, p<0.01$ and $B=0.59 \pm 0.15, t=3.85$, $\mathrm{p}<0.001)$.

The lipid metabolism also changed with the different diets, there was statistical significance between diets and cholesterol levels $(F=4.63$, $\mathrm{df}=2, \mathrm{p}<0.001$ ) being lower on diet 2 and 3 , respectively $(B=-0.24 \pm 0.17, t=-1.37, p=0.17$ and $B=-0.54 \pm 0.17, t=-3.04, p<0.001)$, and triglycerides levels $(F=16.4, d f=2, p<0.001)$ being lower on diet 3 , respectively $(B=-$ $0.45 \pm 0.15, t=-2.93, p=0.001)$. Moreover, it was verified a statistical significance effect between diets on total protein levels $(F=37.9, d f=2$, $p<0.001)$ being higher on diet $3(B=0.40 \pm 0.06$, $t=6.27, p<0.001)$. Likewise, was observed a statistical significance between diets and globulin levels $(F=40.6, d f=2, p<0.001)$ being lower on diet $3(B=-0.47 \pm 0.10, t=-4.39, p<0.001)$ and higher on diet $2(B=0.50 \pm 0.10, t=4.62$, $\mathrm{p}<0.001$ ).

Relationship between biochemical variables and parasites. The effects of type (simple or mixed) infection on serum biochemical variables are presented in table 4 . There was no statistical significance between the type of infection and parasites with albumin levels $(F=0.43, g l=2$, $\mathrm{p}=0.64)$, uric acid levels $(F=1.35, \mathrm{gl}=2, \mathrm{p}=0.26)$ and ALT activity $(F=1.47, d f=2, p=0.23)$. However, a statistically significant difference between the type of infection and parasites with cholesterol levels was observed $(F=7.08, g l=2$, $\mathrm{p}=0.001$ ), being higher in chickens parasitized only by Eimeria spp. $(B=0.67 \pm 0.19, t=3.42$, $\mathrm{p}=0.001$ ) and did not differ for mixed infection by Eimeria spp. and $H$. galinarum $(B=0.20 \pm 0.20$, $\mathrm{t}=0.21, \mathrm{p}=0.19)$, when compared to the uninfected chickens.

A statistically significant difference between infection and parasite with triglyceride levels was observed $(F=8.69, g l=2, p=0.001)$, being lower in the serum of chickens only parasitized by Eimeria spp. $(\mathrm{B}=-0.50 \pm 0.19, \mathrm{t}=-2.57, \mathrm{p}=0.01)$, but without statistical significance for the mixed infection ( $B=0.10 \pm 0.20, t=0.50, p=0.61)$. Statistically significant difference between types of infections and parasites with the total protein levels was verified $(F=13.28, g l=2, p=0.001)$ and globulin $(F=14.65, g l=2, p=0.001)$. The
Relación entre las variables bioquímicas y la dieta. Los resultados de las variables bioquímicas en el suero de las gallinas ponedoras de las tres granjas con dietas diferentes se muestran en la Tabla 3. Hay significancia estadística entre las dietas y los niveles de albúmina $(F=7.62, d f=2$, $\mathrm{p}<0.001$ ) siendo mayores en las dietas 2 y 3 , respectivamente $(B=0.50 \pm 0.13, t=3.74, p<0.01$ y $B=0.38 \pm 0.13, t=2.84, p<0.001)$. También se observó una significancia estadística entre las dietas y los niveles de ácido úrico $(F=23.9, d f=2$, $\mathrm{p}<0.001$ ) siendo mayores en las dietas 2 y 3 , respectivamente $(B=3.42 \pm 0.61, t=5.60, p<0.01$ y $B=3.87 \pm 0.61, t=6.32, p<0.001)$. Otro análisis con significancia estadística se encontró entre las dietas y los niveles ALT $(F=10, d f=2, p<0.001)$ siendo mayores en las dietas 2 y 3 , respectivamente $(B=0.60 \pm 0.15, t=3.90, p<0.01$ y $B=0.59 \pm 0.15$, $t=3.85, p<0.001)$.

El metabolismo de los lípidos también cambió con las diferentes dietas; hubo significancia estadística entre las dietas y los niveles de colesterol $(F=4.63$, $\mathrm{df}=2, \mathrm{p}<0.001$ ) siendo más bajo en las dietas 2 y 3 , respectivamente $(B=-0.24 \pm 0.17, t=-1.37$, $\mathrm{p}=0.17$ y $\mathrm{B}=-0.54 \pm 0.17, \mathrm{t}=-3.04, \mathrm{p}<0.001)$ y los niveles de triglicéridos $(F=16.4, d f=2, p<0.001)$ siendo más bajo en la dieta 3 , respectivamente $(B=-0.45 \pm 0.15, t=-2.93, p=0.001)$. Por otro lado, se verificó un efecto de la significancia estadística entre las dietas sobre los niveles de proteína total $(F=37.9, d f=2, p<0.001)$ siendo mayores en la dieta $3(B=0.40 \pm 0.06, t=6.27, p<0.001)$. Además, se observó una significancia estadística entre las dietas y los niveles de globulina $(F=40.6, d f=2, p<0.001)$ siendo menores en la dieta $3(B=-0.47 \pm 0.10, t=-4.39, p<0.001)$ y mayores en la dieta $2(B=0.50 \pm 0.10, t=4.62$, $\mathrm{p}<0.001)$.

Relación entre las variables bioquímicas y los parásitos. Los efectos del tipo de infección (simple o mixta) en las variables bioquímicas del suero se presentan en la tabla 4 . No hay significancia estadística entre el tipo de infección y de parásitos con los niveles de albúmina ( $F=0.43$, $\mathrm{gl}=2, \mathrm{p}=0.64)$, niveles de ácido úrico $(F=1.35$, $\mathrm{gl}=2, \mathrm{p}=0.26)$ y actividad ALT $(\mathrm{F}=1.47, \mathrm{df}=2$, $\mathrm{p}=0.23$ ). Sin embargo se observó una diferencia estadísticamente significativa entre el tipo de infección y de parásitos con los niveles de colesterol $(F=7.08, g l=2, p=0.001)$, siendo más alto en las gallinas que tenían únicamente el parásito Eimeria spp. $(\mathrm{B}=0.67 \pm 0.19, \mathrm{t}=3.42, \mathrm{p}=0.001)$ y no fue diferente para las infecciones mixtas con Eimeria spp. y H. gallinarum ( $B=0.20 \pm 0.20$, $t=0.1, p=0.19$ ), en comparación con las gallinas no infectadas. 
Table 4. Effect of single infection by Eimeria spp., and mixed infection by genus Eimeria and Heterakis gallinarum on biochemical variables (albumin, uric acid, alanine amiotransferase (ALT), cholesterol, triglycerides, total protein and globulin) in laying hens compared uninfected by gastrointestinal parasites.

\begin{tabular}{lccc}
\hline & \multicolumn{2}{c}{ Mean and standard deviation* } \\
\cline { 2 - 4 } Variables & $\begin{array}{c}\text { Single } \\
\text { infection: } \\
\text { Eimeria spp. }\end{array}$ & $\begin{array}{c}\text { Mixed } \\
\text { infection: } \\
\text { Eimeria } \\
\text { spp. and H. } \\
\text { gallinarum }\end{array}$ & $\begin{array}{c}\text { Control: } \\
\text { Uninfected }\end{array}$ \\
\hline Albumin $(\mathrm{g} / \mathrm{dL})$ & $2.00 \pm 0.40^{\mathrm{a}}$ & $1.91 \pm 0.51^{\mathrm{a}}$ & $2.07 \pm 0.59^{\mathrm{a}}$ \\
Uric acid $(\mathrm{mg} / \mathrm{dL})$ & $5.57 \pm 2.50^{\mathrm{a}}$ & $6.11 \pm 2.85^{\mathrm{a}}$ & $7.09 \pm 2.07^{\mathrm{a}}$ \\
ALT $(\mathrm{U} / \mathrm{L})$ & $22.21 \pm 12.39^{\mathrm{a}}$ & $26.53 \pm 17.96^{\mathrm{a}}$ & $31.08 \pm 18.53^{\mathrm{a}}$ \\
Cholesterol $(\mathrm{mg} / \mathrm{dL})$ & $183.4 \pm 62.3^{\mathrm{a}}$ & $140.3 \pm 43.5^{\mathrm{a}}$ & $89.7 \pm 39.9^{\mathrm{b}}$ \\
Triglycerides $(\mathrm{mg} / \mathrm{dL})$ & $397.5 \pm 117.1^{\mathrm{b}}$ & $651.0 \pm 138.1^{\mathrm{a}}$ & $594.3 \pm 114.7^{\mathrm{a}}$ \\
& & & \\
Total protein $(\mathrm{g} / \mathrm{dL})$ & $4.39 \pm 1.00^{\mathrm{b}}$ & $6.41 \pm 1.81^{\mathrm{a}}$ & $4.77 \pm 1.62^{\mathrm{b}}$ \\
& & & \\
\hline Globulin $(\mathrm{g} / \mathrm{dL})$ & $2.39 \pm 0.96^{\mathrm{b}}$ & $4.50 \pm 1.57^{\mathrm{a}}$ & $2.70 \pm 1.96^{\mathrm{b}}$ \\
\hline
\end{tabular}

* Means followed by equal letter in the same line are not significantly different by Bonferroni's test $(p<0.05)$.

levels of total protein and globulin were higher in chickens infected with mixed infection by parasites $(B=0.26 \pm 0.09, t=2.81, p=0.006$; $B=0.37 \pm 0.15, t=2.32, p=0.02$, respectively)， but no significant difference in single infection by Eimeria spp. $(B=0.10 \pm 0.09, t=0.09, p=0.29$; $B=0.30 \pm 0.16, t=1.78, p=0.08$ ) when compared to the uninfected chickens.

\section{DISCUSSION}

It was found that the laying hens naturally infected with parasites (Eimeria spp and Heterakis gallinarum) presented a change in the levels of blood biochemical variables. Chickens experimentally infected with Ascaridia galli presented decreased seric levels of glucose, total protein, albumin and magnesium (14). Additionally, biochemical studies in an experimental Eimeria necatrix infection in chickens caused a reduction in the levels total protein, albumin and globulin, as well as an elevation in cholesterol concentration in serum (14). Similarly, in our study it was observed a negative correlation between albumin levels and degree of infection by Eimeria (OOPG), i.e., the observation of higher OOPG and lower albumin levels. Also, it was observed an increase of cholesterol in the single infection by Eimeria spp, a similar finding observed by Basith et al (15).

The number of oocysts negatively influenced the serum levels of albumin. It may is explained by the fact that parasitic infections cause
Se observó una diferencia estadísticamente significativa entre el tipo de infección y de parásitos con los niveles de triglicéridos $(F=8.69, g l=2$, $\mathrm{p}=0.001$ ), siendo menor en el suero de las gallinas infectadas solo con Eimeria spp. ( $B=-0.50 \pm 0.19$, $t=-2.57, p=0.01$, , pero sin significancia estadística para las infecciones mixtas $(B=0.10 \pm 0.20, t=0.50$, $\mathrm{p}=0.61$ ). Se verificó la diferencia estadísticamente significativa entre los tipos de infección y de parásitos con los niveles de proteína total $(F=13.28, \mathrm{gl}=2$, $\mathrm{p}=0.001)$ y globulina $(\mathrm{F}=14.65, \mathrm{gl}=2, \mathrm{p}=0.001)$. Los niveles de proteína total y globulina fueron más altos en las gallinas infectadas con infección mixta de parásitos $(B=0.26 \pm 0.09 t=2.81, p=0.006$; $\mathrm{B}=0.37 \pm 0.15, \mathrm{t}=2.32, \mathrm{p}=0.02$, respectivamente), pero no hubo diferencia significativa en infección simple con Eimeria spp. $(B=0.10 \pm 0.09, t=0.09$, $\mathrm{p}=0.29 ; \mathrm{B}=0.30 \pm 0.16, \mathrm{t}=1.78, \mathrm{p}=0.08$ ) cuando se comparó con las gallinas no infectadas.

\section{DISCUSIÓN}

Se encontró que las gallinas ponedoras infectadas naturalmente con parásitos (Eimeria spp y Heterakis gallinarum) presentaron un cambio en los niveles de las variables bioquímicas de la sangre. Las gallinas infectadas experimentalmente con Ascaridia galli presentaron una disminución de los niveles séricos de glucosa, proteína total, albúmina y magnesio (14). Adicionalmente, los estudios bioquímicos en una infección experimental con Eimeria necatrix en las gallinas causaron una reducción en los niveles de proteína total, albúmina y globulina, así como una elevación de la concentración de colesterol en el suero (14). Igualmente, en nuestro estudio se observó una correlación negativa entre los niveles de albúmina y el grado de infección con Eimeria (OOPG), es decir, la observación de mayores OOPG e inferiores niveles de albúmina. Además, se observó un aumento de colesterol en la infección simple por Eimeria $s p p$, un hallazgo similar al observado por Basith et al (15).

El número de ooquistes influenció negativamente los niveles séricos de la albúmina. Esto se puede explicar por el hecho de que las infecciones parasitarias causan enteritis y hemorragia intestinal en las aves, dando lugar a un síndrome de mala absorción que puede poner en peligro el metabolismo de las proteínas $(4,16)$. Los niveles de proteína total y globulina no muestran correlación significativa con el número de ooquistes de Eimeria spp., aun cuando hubo una tendencia $(p=0.08)$ hacia una correlación positiva con globulina. Los niveles de ácido úrico también se influenciaron negativamente por el grado de parasitismo (OOPG). Esto representa la forma principal de excreción variable de los compuestos nitrogenados en las gallinas. Considerando que generalmente la diarrea 
intestine enteritis and bleeding in birds, leading to a malabsorption syndrome that can impair the protein metabolism $(4,16)$. Total protein and globulin levels did not show significant correlation with the number of Eimeria spp. oocysts, although there was a tendency $(p=0.08)$ to a positive correlation with globulin. Levels of uric acid were also negatively influenced by the degree of parasitism (OOPG). It represents the main form of variable excretion of nitrogenous compounds in chickens. Considering that diarrhea is usually caused by these parasites, it may cause an accelerated elimination of nitrogenous compounds (precursors of uric acid), decreasing its synthesis and, consequently, its blood concentration $(5,17)$.

In this study, the ALT activity was lower in hens with larger numbers of oocysts of Eimeria spp. A possible damage to enterocytes caused by this parasite may have impaired the absorption of amino acids and phosphorus, which are important elements in the process of liver ALT biosynthesis (18), this hypothesis can be confirmed starting from analyzes of serum levels of phosphorus, generating a new correlation study. Moreover, changes in serum ALT activity may be related to liver lesion (6), usually increasing its activity. However, we observed an opposite situation where its levels were reduced. Thus, we suggest that the change on ALT activity was not related to tissue damage.

There was no relation between cholesterol and triglyceride (lipid metabolism) with the degree of parasitism (OOPG), but it was observed a reduction in the concentration of triglycerides in animals with single infection by Eimeria. It was already expected since this parasite usually causes intestine lesions $(4,16)$, and therefore, it may lead to malabsorption of fatty acids present in the diet. Additionally, it was observer higher cholesterol levels in laying hens infected with parasites; however, it remained without a plausible explanation.

High levels of total protein and globulin in chickens with mixed infection by Eimeria spp. and $H$. gallinarum may be was related to the immune response against the parasitism (7) It is known that host subjected to parasitic diseases usually activates the humoral and cellular immune responses in order to produce protection against the parasites (19). Our findings in this study suggest that the increase in total protein and globulin were mainly related to $H$. gallinarum infection, since the single infection by Eimeria spp did not change these variables. es causada por estos parásitos, se puede originar una eliminación acelerada de los compuestos nitrogenados (precursores del ácido úrico), disminución de su síntesis y, en consecuencia, de su concentración en la sangre $(5,17)$.

En este estudio, la actividad de la ALT es menor en las gallinas con mayor número de ooquistes de Eimeria spp. Un posible daño a los enterocitos causado por este parásito puede deteriorar la absorción de aminoácidos y fósforo, que son elementos importantes en el proceso de biosíntesis de ALT en el hígado (18); esta hipótesis se puede confirmar a partir del análisis de los niveles séricos de fósforo, generando un nuevo estudio de correlación. Además, cambios en la actividad ALT en el suero pueden estar relacionados con lesiones hepáticas (6), aumentando por lo general su actividad. Observamos, sin embargo, una situación opuesta donde se redujeron sus niveles. Por lo tanto, se sugiere que el cambio en la actividad de la ALT no estaba relacionado con el daño tisular.

No hubo relación entre el colesterol y los triglicéridos (metabolismo de los lípidos) con el grado de parasitismo (OOPG), pero se observó una reducción en la concentración de triglicéridos en los animales con infección simple por Eimeria. Ya se esperaba que este parásito pudiera causar lesiones intestinales $(4,16)$ y, por lo tanto, esto puede conducir a la mala absorción de los ácidos grasos presentes en la dieta. Adicionalmente se observaron altos niveles de colesterol en las gallinas ponedoras infectadas por parásitos; sin embargo, para esto no hay una explicación razonable.

Los altos niveles de proteína total y globulina en las gallinas con infecciones mixtas por Eimeria spp. y $H$. gallinarum pueden estar relacionados con una respuesta inmune contra los parásitos (7). Se sabe que un anfitrión sometido a enfermedades parasitarias normalmente activa las respuestas inmunes humorales y celulares con el fin de producir protección contra los parásitos (19). Nuestros hallazgos en este estudio sugieren que el aumento en la proteína y la globulina total estaba principalmente relacionado con la infección por $H$. gallinarum, ya que la infección simple por Eimeria spp no alteró estas variables.

Se encontró que las gallinas que recibieron las dietas 2 y 3 , con altos niveles de proteína, tuvieron significativamente un contenido de proteína total sérica más alto. Esto se explica ya que existe una estrecha relación entre los niveles nutricionales y la proteína total y la albúmina (20). Del mismo modo, los niveles de ácido úrico fueron también más bajos en las gallinas alimentadas con dietas que contenían cantidades menores de proteína. Este es otro resultado esperado ya que este compuesto se 
It was found that the chickens that received diets 2 and 3, with higher levels of protein had significantly higher seric total protein. It is explained since there is a close relation between nutritional levels, total protein and albumin (20). Similarly, the uric acid levels were also lower in chickens fed with diets with lower protein amounts. It is another expected result, since this compound is synthesized from the amino acid portion of nitrogen, being in the form of nitrogen excretion from birds $(5,18)$. Data of ALT activity showed that diet with lower crude protein resulted in a lower ALT activity. It occurred possibly due to the lower intake of amino acid provided by the diet, as found in broilers at 21 days (21).

The higher levels of blood cholesterol were found in hens under the diet 1 , or with lower levels of energy and crude protein. Our hypothesis is that chickens receiving this diet synthesized cholesterol more intensely, especially because the three diets evaluated were prepared from ingredients of vegetable origin, and therefore, cholesterol free. Another possible explanation is the use of oils rich in unsaturated fatty acids and polyunsaturated fatty acids in the diets 2 and 3, which were more energetic. According of Murray et al. (17) these fatty acids act reducing cholesterol ( $L D L$ ) by increasing the catabolism of $\mathrm{LDL}$, the major atherogenic lipoprotein protein. Additionally, triglyceride levels were higher in the diet with greater energy levels. However, the levels of this variable were closely related to the time of blood collection, because the lipid metabolism in laying hens is very unstable, since the yolk is composed mainly of fats.

Based on the results it can be concluded that infection with parasites influence on serum biochemical variables in laying hens. The reduction of some biochemical variables may be related to intestinal damage due to the parasitism by Eimeria spp, a well-known factor described in the literature, and therefore, contributing to a less absorption of nutrients. The increase of total protein and globulin were suggestive of immune response activation against $H$. gallinarum. Finally, this study also confirmed that the diet has a direct effect on biochemical variables. Therefore, to avoid losses in production emphasize the importance of the use of coccidiosis control programs (22). sintetiza a partir de la proporción de nitrógeno en los aminoácidos, en forma de excreción de nitrógeno por parte de las aves $(5,18)$. Los datos de actividad de la ALT mostraron que la dieta con baja proteína cruda dio como resultado una menor actividad de la ALT. Esto ocurrió posiblemente debido a la menor ingesta de aminoácidos proporcionada por la dieta, como se observó en los pollos de engorde a los 21 días (21).

Se encontraron altos niveles de colesterol en la sangre en las gallinas sometidas a la dieta 1 o menores niveles de energía y proteína cruda. Nuestra hipótesis es que las gallinas que recibieron esta dieta sintetizaron el colesterol más intensamente, especialmente porque las tres dietas evaluadas se prepararon con ingredientes de origen vegetal $y$, por lo tanto, libre de colesterol. Otra posible explicación es el uso de aceites ricos en ácidos grasos insaturados y polinsaturados en las dietas 2 y 3 , que fueron más energéticos. De acuerdo con Murray et al (17), estos ácidos grasos actúan reduciendo el colesterol (LDL) mediante el aumento del catabolismo del LDL, la principal proteína de las lipoproteínas aterogénicas. Además, los niveles de los triglicéridos fueron más altos en las dietas con mayores niveles de energía. Sin embargo, los niveles de esta variable estaban estrechamente relacionados con el momento de la toma de sangre, debido a que el metabolismo de los lípidos en las gallinas ponedoras es muy inestable, ya que la yema de huevo se compone principalmente de grasas.

Con base en los resultados se puede concluir que la infección con parásitos influye en las variables bioquímicas en el suero de las gallinas ponedoras. La reducción de algunas variables bioquímicas puede estar relacionado con daños intestinales debido al parasitismo por Eimeria spp, un factor bien conocido y descrito en la literatura, y que además contribuye a una menor absorción de los nutrientes. El incremento de la proteína total y la globulina sugieren la activación de la respuesta inmune contra la $H$. gallinarum. Por último, este estudio también confirmó que la dieta tiene un efecto directo en las variables bioquímicas. Por lo tanto, para evitar pérdidas en la producción se hace énfasis en la importancia de implementar programas de control de la coccidiosis (22). 


\section{REFERENCES}

1. Cardozo SP, Yamamura MH. Parasites in freerange chickens system in Brazil. Semina: Ciênc Agr 2004; 25(1):63-74.

2. Abbas RZ, Munawar SH, Manzoor Z, Iqba Z, Khan MN, Saleemi MK, Zia MA, Yousaf A. Anticoccidial effects of acetic acid on performance and pathogenic parameters in broiler chickens challenged with Eimeria tenella. Pesq Vet Bras 2011(1); 31(2):99-103.

3. Dahmer J, Tochetto C, Franco CB, Mahl D, Da Silva AS, Monteiro SG. Espécies de Eimeria em frangos de corte. Rev Cient Elet Med Vet 2008; 6(2):1-6.

4. Persia ME, Young EL, Utterback PL, Parsons CM. Effects of dietary ingredients and Eimeria acervulina infection on chick performance, apparent metabolizable energy, and amino acid digestibility. Poult Sci 2006; 85(1):48-55.

5. Kaneko JJ, Harvey JW, Bruss ML. Clinical Biochemistry of Domestic Animals. 6ed., Waltham, Academic Press 2008; 928p.

6. Grunkemeyer VL. Advanced diagnostic approaches and current management of avian hepatic disorders. Vet Clin N Am: Exot Anim Pract 2010; 13(3):413-427.

7. Abdel-Fattah SA, El-Sanhoury MH, El-Mednay NM, Abdel-Azeem F. Thyroid activity, some blood constituents, organs morphology and performance of broiler chicks fed supplemental organic acids. Int J Poult Sci 2008; 7(2):215-222.

8. Martín-Castillo A, Castells MT, Adánez G, Polo MT, Pérez BG, Ayala I. Effect of atorvastatin and diet on non-alcoholic fatty liver disease activity score in hyperlipidemic chickens. Biomed Pharmacother 2010: 64(3):275-281

9. Adamu M, Boonkaewwan C, Gongruttananun $\mathrm{N}$, Vongpakorn M. Hematological, biochemical and histopathological changes caused by coccidiosis in chickens. Kasetsart J 2013; 47(3):238-246.

10. Soriano VS, Sá J, Rampazzo-Junior HP, Campigotto G, Mattiello TL, Grosskopf HM, Tonin AA, Da Silva AS. Biochemical variable levels of newborn lambs under extensive rearing system. Comp Clin Pathol 2014: 23(5):1-5.

11. Matterson LD, Potter LM, Stutz MW. The metabolizable energy of feed ingredients for chickens. Storrs: Connecticut University of
Connecticut, Agricultural Experiment Station 1965; 7(1):11-14.

12. Van Soest PJ, Robertson JB. Chemical and physical properties of uncommon feeedstuff's. Proc Cornell Nutrition Conf Ithac, NY. 1976; 12(1):1-45.

13. Rice WR. Analizyng tables of statistical tests. Evol 1989; 43(2):223-225.

14. Ayub AM, Prasad $H$, Lyngdoh WM, Das G, Prava M, Victoria CKH, Tolenkhomba TC, Singh YD, Ralte L, Inaotombi DL. Biochemical profile of broiler chickens experimentally infected with Ascaridia galli. Indian J Anim Res 2013; 47(1):1-17.

15. Basith SB, Rajavelu G, Manohar BM. Biochemical studies in experimental Eimeria necatrix infection in chickens. J Indian Vet 1998; 75(7):876-878.

16. Idris $A B$, Bounous DI, Goodwin MA, Brown J, Krushinskie EA. Lack of correlation between microscopic lesion scores and gross lesion scores in commercially grown broilers examined for small intestinal Eimeria spp. coccidiosis. Avian Dis 2007; 41(4):388-391.

17. Murray RK, Botham KM, Kennelly PJ, Rodwell VW, Anthony-Weil P. Harper Bioquímica ilustrada. 29ed., Rio de Janeiro, McGraw-Hill Brasil; 2014.

18. Jaensch S. Diagnosis of avian hepatic disease. Sem Avian Exot Pet Med 2005; 9(1):126-135.

19. Galha V, Bondan EF, Lallo MA. Relação entre imunossupressão e coccidiose clínica em frangos de corte criados comercialmente. Rev Inst Ciênc Saúde 2008; 26(4):432-437.

20. Dunbar MR, Gregg MA, Crawford JA, Giordano MR, Tornquist SJ. Normal hematologic and biochemical values for prelaying greater sage grouse (Centrocercus urophasianus) and their influence on chick survival. J Zoo Wildl Med 2005; 36(3):422-429.

21. Vieites FM, Moraes GHK, Albino LFT, Rostagno HS, Rodrigues AC, Silva FA, Atencio A. Balanço eletrolítico e níveis de proteína bruta sobre parâmetros sanguíneos e ósseos de frangos de corte aos 21 dias de idade. Rev Bras Zootec 2004; 33(6):1520-1530.

22. Chapman HD. Rotation programmes for coccidiosis control. Int Poult Product 2007; 15(1):7-9. 